\title{
Tangence
}

\section{L'architecte du lisible}

\section{Lecture de L'écologie du réel de Pierre Nepveu}

\section{Anne-Marie Clément, Robert Dion et Frances Fortier}

Numéro 51, mai 1996

Paradigmes critiques

URI : https://id.erudit.org/iderudit/025908ar

DOI : https://doi.org/10.7202/025908ar

Aller au sommaire du numéro

Éditeur(s)

Tangence

ISSN

0226-9554 (imprimé)

1710-0305 (numérique)

Découvrir la revue

Citer cet article

Clément, A.-M., Dion, R. \& Fortier, F. (1996). L'architecte du lisible : lecture de L'écologie du réel de Pierre Nepveu. Tangence, (51), 123-143.

https://doi.org/10.7202/025908ar d'utilisation que vous pouvez consulter en ligne.

https://apropos.erudit.org/fr/usagers/politique-dutilisation/ 


\section{L'architecte du lisible. Lecture de L'écologie du réel de Pierre Nepveu*}

Anne-Marie Clément, Robert Dion et Frances Fortier

Sans doute la question des paradigmes critiques a-t-elle partie liée avec la prolifération des gloses et des interprétations divergentes - de telle sorte que le pluriel de l'intitulé de la présente livraison de Tangence, en plus de souligner la multiplicité des matrices ${ }^{1}$ d'où émanent les discours critiques pratiqués aujourd'hui, paraît indiquer qu'il n'y a de paradigmes perceptibles que s'il en est plusieurs, qui s'interpellent, se répondent et se contestent, et que seul un improbable discours totalitaire pourrait se penser hors paradigme. Tout paradigme n'est donc envisageable que parmi des paradigmes concurrents, et l'hétérogénéité leur est en quelque sorte consubstantielle, comme en témoigne d'ailleurs l'étymologie du mot $^{2}$. Mais parallèlement à l'hétérogénéité des paradigmes, il faut aussi parler des paradigmes de l'hétérogène, qui semblent actuellement dominer tant dans les discours esthétiques (Barth, Robin, etc.) que dans la critique. Cette question de l'hétérogénéité et spécialement de l'hétérogénéité énonciative est celle qui nous préoccupe dans la recherche que nous menons en ce moment sur la critique québécoise depuis $1980^{3}$.

Nous visons dans cette recherche à relever les formes de subjectivité/intersubjectivité et d'interdiscursivité repérables dans les

* Cette étude appartient à une série consacrée aux Dispositifs énonciatifs du discours critique québécois depuis 1980. Ce projet est subventionné par l'Université du Québec à Rimouski.

1 Pierre Ouellet rappelle que le terme "matrice" est chez Kuhn l'autre nom pour "paradigme"; voir "la Notion de paradigme : de l'histoire des sciences à l'histoire littéraire", dans Clément Moisan (dir.), L'bistoire littéraire: théories méthodes/pratiques, Québec, Presses de l'Université Laval, 1989, p. 14.

2 "Para" et "deiknumi ", c'est-à-dire "montrer" quelque chose "en comparaison"; voir encore Ouellet, op. cit., p. 10.

3 Pour une description de ce projet, voir Robert Dion: "Dispositifs énonciatifs du discours critique québécois depuis 1980 ", dans Lucie Bourassa (dir.), La discursivité, Québec, Nuit blanche éditeur, 1995, p. 185-202. Un court extrait de cette description est repris ci-après. 
ouvrages de critique parus au Québec après $1980^{4}$, époque où prolifèrent les métadiscours qui font pièce aux théories structurosémiotiques en proposant des formes inédites, ostensiblement subjectives, qui ont pu être dites postmodernes. Il va sans dire que ces formes sont construites par et dans l'énonciation, bien qu'elles échappent, en vertu des exigences interdiscursives mêmes du "formulaire scientifique " en vigueur dans les études littéraires depuis au moins la décennie soixante, à la seule instance productrice du discours. Nous sommes ainsi conduits à poser la question suivante: comment un discours critique prétendument subjectif, voué à l'expression d'une pensée individuée ou voulue telle, peut-il néanmoins manifester un caractère éminemment intersubjectif et interdiscursif, c'est-à-dire donner à voir l'interaction de sujets de connaissance et se situer au croisement d'une panoplie de discours, celui de l'œuvre, du mouvement ou de la période commentés, certes, mais également ceux de l'institution littéraire, des réceptions antérieures, de la théorie, etc.? Bref, comment une parole critique qui s'envisage elle-même comme singulière arrive-t-elle à émerger de cet entrelacs discursif, à se dégager de la foncière transsubjectivité où est pourtant engluée toute énonciation?

La mise au jour et la description d'un paradigme de l'hétérogénéité (qui appartient, on l'a vu, à une aire discursive elle-même partagée entre des paradigmes hétérogènes) passe par l'analyse des stratégies énonciatives d'un certain nombre d'ouvrages de critique littéraire parus au Québec durant la période en cause. Pour le présent article, et aux fins d'une première expertise, nous n'avons retenu qu'un seul ouvrage, L'écologie du réel de Pierre Nepveu ${ }^{5}$. Nous l'avons choisi d'une part parce que, en tant qu'essai constitué d'articles déjà parus auparavant, il procède d'une dialectique entre diversité et unité, d'autre part en raison de la hauteur de sa visée: embrasser l'ensemble de la littérature québécoise contemporaine et en entamer la relecture.

4 Nous avons retenu les ouvrages et non les articles parce que les premiers, dans la mesure où ils reprennent souvent les seconds et les remettent en contexte dans une composition de plus grande envergure, opèrent déjà une paradigmisation.

5 Pierre Nepveu, L'écologie du réel. Mort et naissance de la littérature québécoise contemporaine, Montréal, Boréal, coll, "Papiers collés", 1988. Les références à cet ouvrage seront désormais signalées par le folio entre parenthèses. 
La critique a accueilli favorablement L'écologie du réel ${ }^{6}$. La connaissance intime qu'a Nepveu de la littérature québécoise contemporaine ainsi que ses qualités de lecteur ont été largement remarquées. On lui reconnaît le mérite de proposer une relecture féconde, offrant une vision intégrée des ouvres de la tradition québécoise. Cette relecture se fait essentiellement à la lumière du paradoxe que Nepveu présente d'entrée de jeu, paradoxe issu de la coprésente des enjeux de la modernité (discours du désastre, de la solitude, de la fin) et des enjeux de la fondation du pays (discours de la vitalité, du commencement). Quant aux principales réserves, elles concernent le manque de précision et l'absence de regard critique à l'endroit de certains des concepts utilisés (écologie, catastrophisme, littérature postquébécoise).

Le cas de lauteur est au surplus intéressant: à cheval entre deux générations, coopté tant par ses aînés de l'Hexagone que par ses contemporains de la "nouvelle écriture", professeur et écrivain polygraphe (à la fois poète, romancier, essayiste et critique), Pierre Nepveu occupe une position charnière dans l'institution littéraire québécoise, et sa polyvalence ne peut que se refléter dans son discours, fondé sur cet ensemble d'expériences et de savoirs divers. Nous avons pour notre propos retenu deux axes de réflexion: l'un sur la relation de l'énonciateur à son propre discours (discours au statut problématique, à mi-chemin entre la critique universitaire et l'essai), l'autre sur le lien entre le discours de l'instance énonciatrice et celui d'autrui, lieu de manifestation privilégié du paradigme de l'hétérogène.

6 Voici la liste des principales recensions critiques qui ont suivi la parution de cet ouvrage: Réjean Beaudoin et Robert Melançon, "Chronique d'une mort différée", Libertê, no 183 , juin 1989, p. 138-143; Thierry Horguelin, «Le conflit insoluble de la modernité", Spirale, no 87, avril 1989, p. 8-9; Robert Major, "Papiers collés, papiers à lire", Voix et images, n ${ }^{\circ} 42$, printemps 1989 , p. 498 503; Agnès Whitfield, "Pour une littérature post-québécoise", Lettres québécoises, $\mathrm{n}^{\circ} 53$ (1989), p. 45-46. Il est également question de l'ouvrage de Nepveu dans Michel Biron, "Dépayser la littérature québécoise", Spirale, $n^{\circ} 100$, octobre 1990 , p. 5-6; François Dumont, "L'essai littéraire québécois des années quatre-vingt: la collection "Papiers collés" ", Rechercbes sociographiques, vol. XXXIII, $\mathrm{n}^{\circ} 2,1992$, p. 323-335. 
126

\section{Les marques de la subjectivité}

Dans un premier temps, nous voulons examiner la coexistence, chez Nepveu, de deux types de discours, celui de la critique universitaire et celui de l'essai - soit, respectivement, de l'essai cognitif et de l'essai-méditation, selon la typologie de Marc Angenot ${ }^{7}$. Ces deux discours impliquent des attitudes énonciatives contradictoires, l'une favorisant l'effacement de l'énonciateur et l'autre montrant le sujet écrivant, support d'une pensée individuée. Ce type d'hybridation entre des discours n'est pas en soi exceptionnel, loin de là, mais nous voulons voir comment s'articulent ces deux postures énonciatives, comment s'effectue le passage de l'une à l'autre, s'il y a des lieux du texte privilégiés par l'une ou par l'autre, si l'une est subordonnée à l'autre, si la présence de l'une éclipse momentanément l'autre, etc. L'examen de l'utilisation des différents pronoms je, nous, on, de la formulation interrogative et des parenthèses mettra en lumière les modulations du rapport de l'énonciateur à son discours et précisera les formes de son engagement.

L'ouvrage, selon la politique établie de la collection "Papiers collés", regroupe des articles publiés auparavant. Dans ce nouveau contexte, les articles repris changent quelque peu de statut, car, bien que le contenu soit sensiblement le même, la situation d'énonciation est différente. Cette mise en perspective participe au sens à accorder au texte. Si l'ouvrage dans son ensemble est considéré comme un essai, il est probable que certains des chapitres, parus sous forme d'articles dans des revues littéraires spécialisées, aient d'abord été considérés comme des analyses relevant de la critique littéraire. Dans une certaine mesure, cette réorganisation du point de vue de l'énonciation ajoute un nouveau palier où se lit le travail de liaison entre des textes d'abord présentés isolément; ces derniers ont subi des modifications plus ou moins importantes ${ }^{8}$. Nepveu a également produit plusieurs chapitres

7 Voir La parole pampblétaire, Paris, Payot, 1982.

8 Par exemple, le chapitre IX, intitule "Ontologie et Écologie", remanie et fusionne deux articles déjà parus, à savoir "Trois romans de Nicole Brossard: une histoire au présent ", Incidences, vol. IV, ${ }^{\text {os }} 2-3$, mai-décembre 1980 , p. 129-138, et "Nicole Brossard: notes sur une écologie", La nouvelle barre du jour, $\mathrm{n}^{\text {os }} 118-119$, novembre 1982 , p. 139-144. Outre l'important travail de réécriture, il faut noter le traitement différent de la notion d'écologie. Ainsi, dans l'article de 1982, Nepveu écrivait, à propos de Brossard: "À travers le 
inédits ( $\mathrm{l}$, IV, VII, X, XII, "Conclusion") qui viennent s'insérer dans ce projet global, projet dont les tenants et les aboutissants sont présentés dans l'"Avertissement" (p. 9-12) et la "Conclusion" (p. 211-220). Dans l' Avertissement", le critique signale que l'ouvrage est le fruit d'une réflexion qui s'étend sur une période d'environ dix ans pendant laquelle un certain nombre des essais maintenant regroupés ont été publiés (on retrouve les références bibliographiques précises dans la "Note bibliographique" en fin de volume). Par conséquent, la démarche de Nepveu se donne comme un projet unique, issu d'une réflexion spécifique, et non comme un collage d'analyses produites antérieurement et regroupées sous une même thématique. C'est davantage en tant qu'essayiste que l'auteur se met en scène dans cet "Avertissement", ce que l'énonciation à la première personne vient appuyer; or ce type d'énonciation ne sera pas conservé tout au long de l'ouvrage.

L'examen des pronoms utilisés dans L'écologie du réel met en évidence les diverses postures énonciatives adoptées par l'auteur. Considérant l'ampleur du texte, il va de soi que notre lecture ne saurait s'attacher à l'étude détaillée des occurrences; nous croyons cependant que le texte suggère ses propres regroupements qui facilitent la saisie de ces informations. Ainsi, dès le premier chapitre, chapitre introductif, le je est laissé de côté au profit de formulations plus impersonnelles. Omniprésent dans l' Avertissement" (et également dans la "Conclusion", qui revient sur les objectifs fixés au départ), ce pronom va se rencontrer rarement mais régulièrement au fil des chapitres et assumer principalement la fonction de régie; c'est le je qui renvoie le lecteur à un ailleurs dans l'ouvrage ${ }^{9}$ ou encore qui agit comme organisateur de l'ordre

processus de réduction et de recueillement à l'ouvre dès 1970 dans son écriture, c'est l'élaboration concrète d'une écologie qui a toujours été en cause. Une écologie au sens où Gregory Bateson emploie ce terme dans Vers une écologie de l'esprit, c'est-à-dire au sens d'une vision systémique de l'ensemble corps-esprit-monde, système auto-régulé à plusieurs niveaux." (op. cit., p. 142); dans L'écologie du réel, le critique convoque la notion d' "écologie de l'esprit" de Bateson - sans toutefois la définir - pour dénoncer, paradoxalement, le fait que "la notion d'écologie, et toute la pensée systémique qui s'y rattache, n'a guère été pensée par les intellectuels québécois " (p. 153).

9 "Comme je tente de le montrer plus loin dans cet ouvrage" (p. 25); "je me propose d'y revenir plus tard" (p. 45); "j'y reviendrai, en temps et 
du discours ${ }^{10}$. La première personne est en outre présente dans des locutions du type "me semble-t-il", "à mon avis" ${ }^{11}$; ces modalités précisent la nature de l'adhésion du locuteur à son énoncé et signalent la "présence pensante" de l'essayiste dans un discours qui, le plus souvent, se tait sur l'énonciateur. On observe quelques variations dans la fréquence d'utilisation du je au gré des chapitres, ce qui invite à mettre en relation l'usage de ce pronom avec les autres formes d'énonciation utilisées. Par exemple, le je est absent du premier chapitre ou, plus exactement, il se trouve relégué aux notes situées à la fin de l'essai. Cette mise à l'écart du sujet et de sa subjectivité fait place à une énonciation impersonnelle et à une présentation plus "objective" des énoncés: un lieu neutre se dégage, permettant la mise en place de notions et d'hypothèses qui semblent s'énoncer d'ellesmêmes ${ }^{12}$.

C'est également dans ce premier chapitre que s'instaure un nous qui, sans être clairement assigné à un ou à des actants, s'associe assez aisément soit à la communauté constituée par l'énonciateur et ses énonciataires, soit à la collectivité québécoise dans son ensemble, collectivité associée au destin de cette littérature québécoise et post-québécoise, ou encore aux deux à la fois ${ }^{13}$. Ce pronom conserve une certaine imprécision qui ne change rien au fait qu'il est responsable de l'émergence d'un espace collectif réunissant le locuteur et des allocutaires. Dès le deuxième chapitre, le je-locuteur réapparaît dans le texte, mais discrètement: au début du chapitre pour assurer la régie et préciser sa position (ce que Nepveu ne fait pas fréquemment: peutêtre uniquement lorsqu'il se sent en étroite connivence avec certains auteurs; ici, il s'agit de Saint-Denys Garneau, à qui il va consacrer plusieurs pages de son livre), ensuite sous la forme du "me semble-t-il" mentionné ci-haut. Une autre intervention

lieu" (p. 77); "dont j'al parlé» (p. 159); “j’ai mentionné à cet égard" (p. 174); "j'ai parlé plus tôt " (p. 174).

10 "Il est temps d'en revenir à ma question. (p. 113); “je propose de relire deux romans" (p. 159); "je propose que nous revenions" (p. 181).

11 Par exemple, aux pages $28,41,88,137,176,192$ et 210.

12 "Une des hypothèses de ce livre est" (p. 16).

13 Jean-Michel Adam parlerait dans le premier cas de référence discursive et dans le second, de référence situationnelle. Pour cette question des ancrages énonciatifs, voir Éléments de linguistique textuelle, Liège, Mardaga, 1990, p. 101. 
directe se produit vers la fin du chapitre ${ }^{14}$; les autres signes déictiques renvoyant à la situation d'élocution demeurent rares ${ }^{15}$.

Il en sera ainsi dans la majorité des chapitres: présence d'un je ponctuel maitre de l'organisation de son discours; présence occasionnelle d'un nous qui renvoie à la communauté plus ou moins élargie et par lequel s'établit une connivence entre le locuteur et son vis-à-vis, tous deux inclus dans cette communauté du nous. De fait, la lecture de Nepveu est à maints endroits une lecture accompagnatrice très proche du tutorat caractéristique de l'enseignement: au chapitre deux, par exemple, après avoir suscité l'intérêt par un astucieux "faux" poème ${ }^{16}$ et par une série d'interrogations, il enchaîne sur un "Considérons donc" qui va tout à fait dans le sens d'un certain soutien pédagogique (p. 26).

Dans le texte d'une conférence donnée lors d'un colloque sur Crémazie et Nelligan (dans ce cas particulier, nous nous référons au texte original de l'allocution) ${ }^{17}$, Nepveu précise le contenu de ce nous: «le lieu où nous y projetons, écrivains, lecteurs, critiques, professeurs, étudiants et autres, des significations, des symboles, des mythes" ${ }^{18}$. Certes, ce nous sert à décrire l'auditoire en question, mais l'auteur prend soin d'en donner une définition très englobante. Notons, de plus, que la complicité ne concerne pas uniquement le conférencier et ses auditeurs, mais aussi les écrivains en cause; elle se définit à partir du "lieu imaginaire de la littérature québécoise", lieu qui inclut de toute évidence Nelligan et Crémazie, mais aussi l'auditoire, le conférencier et son propre discours (qu'il refuse de considérer comme extérieur à cet imaginaire: «j'en fais partie, comme vous tous, à des titres divers, et déjà par le simple fait de participer à un colloque où sont juxtaposés les noms de Crémazie et Nelligan ${ }^{19}$ ). Au sujet de la

14 "Ces références me paraissent particulièrement éclairantes pour Garneau " (p. 40).

15 Notons le déictique "ici": "on soupçonne ici un paradigme" (p. 30).

16 Mise en scène qui montre à l'évidence la grande latitude dont jouit le locuteur quant à la façon de rapporter le discours d'autrui.

17 "Crémazie et Nelligan: l'exil comme métaphore", dans Réjean Robidoux et Paul Wyczynski (dir.), Crémazie et Nelligan, Montréal, Fides, 1981, p. 132140; cet article est à l'origine du chapitre III du présent ouvrage.

18 Ibid., p. 132.

19 Loc. cit. Voici l'ensemble du paragraphe d'où est extraite cette citation: "Dans cette totalité imaginaire, Crémazie et Nelligan occupent une place, et c'est cette place que je voudrais tenter d'examiner, tout en sachant fort bien 
130

communauté du nous, Nepveu ajoutera au chapitre XI de L'écologie du réel: "la communauté des anonymes, et des solitudes, unie par une douleur, un manque, un désir de parole, un "nous" fantasmatique dont on ne peut pourtant dire qu'il soit tout à fait irréel" (p. 190) (c'est nous qui soulignons).

Mis à part ces quelques interventions, l'énonciation est souvent prise en charge par un on ou un $i l$ impersonnel, ou alors ce sont les notions et les objets d'étude qui s'énoncent par euxmêmes. Par exemple, à la seule page 19, on lit que la "littérature "québécoise", elle, transforme, ou veut transformer, la réalité"; que "les infinies possibilités de développement du moi "s'affirment dans "une sorte de mythe faustien"; que "l'exigence d'une perpétuelle et infinie destruction" se pose dans la modernité; que la "transformation du réel" suppose une "habitation radicale du présent"; etc. Le discours semble dès lors "soumis à la force contraignante des idées" ${ }^{20}$; celles-ci ne sont plus les idées "personnelles " d'un auteur, elles s'imposent par la force de l'évidence. En donnant l'impression d'aller de soi, d'être objectif, ce type de formulation est une autre façon de valider le propos; l'autorité discursive remplace, en quelque sorte, la parole autoritaire ${ }^{21}$. Elle laisse, du reste, une plus grande liberté d'adhésion de la part de l'allocutaire, d'où, peut-être, la nécessité de raviver régulièrement la connivence de ce dernier en recourant à des formes plus personnelles d'énonciation.

En plus du système des pronoms, nous voudrions présenter rapidement quelques-uns des aspects par lesquels Nepveu affiche tout particulièrement sa subjectivité : la formulation interrogative, la modalisation du discours d'escorte et l'usage des parenthèses.

que le mot "place" ne peut avoir ici la signification nette et précise qu'il a dans une expression comme "place de stationnement". Nous ne sommes pas dans l'espace, nous sommes dans l'imaginaire, où rien n'est fixe ni définitif, où les significations ne cessent de se reformer. Et puis, il y aurait une terrible prétention positiviste à vouloir mettre son propre discours tout à fait à l'extérieur de cet imaginaire: j'en fais partie, comme vous tous, à des titres divers, et déjà par le simple fait de participer à un colloque où sont juxtaposés les noms de Crémazie et Nelligan.

20 Angenot, op. cit., p. 4.

21 Ainsi, on retrouve au premier chapitre (p. 13-24) les tournures syntaxiques suivantes: "il y a là un fait bien connu "; "il ne suffit pas ici "; "il faut plutôt "; "comme on pouvait s'y attendre"; "comme toujours"; "il est impensable"; "dès lors, il faut "; "il s'agit bien"; "il est vrai que *. 
Deux de ces aspects reposent sur des marques graphiques (le point d'interrogation et les parenthèses) et ont été repérés à partir d'un balayage du texte.

Nepveu utilise beaucoup la forme interrogative. Les trois premiers paragraphes du premier chapitre, ainsi que les deux premiers paragraphes (soit avant et après le "faux" poème de Garneau) du deuxième, commencent par une ou des interrogations qui précisent, à l'intention du lecteur, la nature du questionnement de l'ouvrage ou du chapitre. En plus d'exposer les grandes questions qui sous-tendent la réflexion de l'auteur, cette stratégie énonciative instaure une situation de dialogue et peut être utilisée pour tracer le profil de l'allocutaire à l'intérieur du discours. Dans L'écologie du réel, l'interrogation est presque toujours immédiatement suivie de sa réponse ${ }^{22}$; de sorte que le locuteur paraît être celui qui a la possibilité d'instruire un allocutaire qui est participant de cet échange - n'est-ce pas sa question que le locuteur a devinée, transcrite, et à laquelle il répond? C'est également sous une forme interrogative que certains jugements ou idées ayant cours subissent une "réévaluation" de la part du locuteur; dans ces cas, la question est en général posée à partir d'un groupe nominal et aucun signe ne permet de lui attribuer un énonciateur ${ }^{23}$; on peut néanmoins déduire qu'il s'agit du discours de l'Autre, discours que Nepveu utilise pour inscrire sa propre position, souvent discordante (ce discours anonyme de l'Autre est à distinguer de celui du pair admiré, co-énonciateur savant ou écrivain). La formulation interrogative favorise donc le

22 ‘Pourquoi commencer ce parcours [...]? Sans doute par ce qu'il est [...] "notre premier poète de la modernité".(p. 25); "que manquait-il? Essentiellement : une notion centrale" (p. 46); "De quelle réalité parle ce discours du manque [...]? Sans doute du lieu même de son discours" (p. 60); "comment Grandbois pouvait-il nourrir une prise de conscience [...]? D'abord par la force de son style" (p. 66); "Mais toute narrativité, justement, se trouve-t-elle abolie? Non, et ce non a une double portée" (p. 96); "Que conclure de cette déroute? D'un côté, la poésie se donne comme transcendance absolue. (p. 131); "Quelle communauté? Mais celle des exilés" (p. 190); "Qu'est-ce que cela veut dire? Il va de soi que la littérature se pose ici contre cette "nostalgie de l'imperméabilité" (p. 209).

23 "Poèmes de jeunesse, bientôt reniés par une pratique formaliste? C'est trop vite dit." (p. 59); "Métaphores maladroitement ornementales? Mais elles disent quelque chose sur le pouvoir d'envoûtement" (p. 64); "Échec de la poésie? Mais Chamberland [...] est justement en train de dire" (p. 75); "Une cérémonie des adieux? Rien n'est moins sûr." (p. 77); "Éternel retour du même? Pas vraiment. " (p. 184). 
déploiement d'une stratégie donnant l'occasion au locuteur de se démarquer du discours d'autrui, et facilite l'élaboration d'une tactique rhétorique propre à susciter l'intérêt de l'allocutaire ${ }^{24}$.

L'attitude d'un locuteur par rapport au discours qu'il cite peut varier. Il est en mesure d'introduire le discours d'autrui avec des verbes "neutres" et de se constituer ainsi en tant que locuteur objectif, ou encore d'adopter la position inverse qui consiste à manifester sa subjectivité en se prononçant sur ce discours. Lorsque les propos sont rapportés en style direct, il y a effectivement rencontre de deux discours issus de deux locuteurs. Le locuteur citant donne donc la version "authentique " du discours de l'autre locuteur; il doit toutefois être également en mesure de faire entendre son propre discours, d'offrir sa lecture. Cela est encore plus crucial dans un texte où abondent les citations. Chez Nepveu, on trouve fréquemment des "évaluations" positives du discours rapporté ${ }^{25}$ - discours archivé et, de ce fait, pratiquement accrédité d'office. En portant un jugement sur le discours cité, le critique signale sa position de locuteur principal, il s'approprie le discours cité et le soumet aux visées de son propre discours; le fait que le jugement soit généralement élogieux n'étonne pas dans un texte qui ne cherche pas la polémique et dont l'avancée s'appuie, comme nous le verrons, sur le cumul de témoignages concordants. De plus, il faut noter qu'à travers ces

24 Cette tactique est à l'œuvre dans cet exemple où, à l'intérieur de deux paragraphes, une succession de questions relance le discours : "que se passe-t-il? [...] Or qu'est-ce que le réel? [...] Mais quel roman? Balzac?" (p. 105).

25 Voici une liste d'exemples où le commentaire de Nepveu exprime son attitude à l'endroit du discours rapporté: "Leclerc, dans une formule fulgurante" (p. 16); "comme le dit si bien Miron" (p. 17); "depuis les pages irréfutables consacrées par Gilles Marcotte" (p. 28); "Ces références [Adorno, Lotman, Pasternak] me paraissent particulièrement éclairantes" (p. 40); "Comme l'a écrit Yvon Rivard dans un texte d'une remarquable lucidité" (p. 40); "Comme le dira si bien Hubert Aquin" (p. 66); "il est significatif de voir Jacques Brault (p. 67); "comme le fait très justement observer André Brochu" (p. 101); atel est l'un des plus beaux vers" (p. 101); "comme l'a bien montré Gilles Marcotte " (p. 102); "selon la superbe formule de Platon" (p. 119); "ce monologue [de Derrida] tout à fait remarquable" (p. 157); "les idées riches et fort complexes de Mary Douglas sur le rituel" (p. 168); "texte [de Belleau] qui, dans sa remarquable ambiguiité" (p. 182); "qu'Anne Hébert avait déjà superbement mise en scène dans son poème" (p. 183); "comme l'a superbement montré Christine Buci-Glucksmann" (p. 193). On voit se dessiner ici ce qui ressemble à un palmarès - qui désigne maintes figures dominantes/ "consacrantes " de la sphère de la critique québécoise. 
marques d'appréciation, Nepveu laisse transparaître un lecteur sensible aux idées et aux mots du texte, au "si bien dire" de l'écrivain et de l'écriture, à la force des idées et au "plaisir du texte".

Le recours pléthorique aux parenthèses - il y en a un nombre impressionnant tout au long de l'essai, pratiquement à toutes les pages ${ }^{26}$ — témoigne encore de la subjectivité de l'essayiste. Elles renferment divers types d'informations et visent principalement à préciser le sens ou la portée d'un $\operatorname{mot}^{27}$, à donner des exemples ${ }^{28}$, à commenter ${ }^{29}$. Les parenthèses constituent chez Nepveu un lieu privilégié du métadiscours; le critique y glose sur

26 Précisons cependant que la répartition n'est pas égale dans les différents chapitres: par exemple, les 15 pages du chapitre XI en contiennent environ 5 , alors qu'il y en a autour de 50 pour les 25 pages du chapitre $X$.

27 "La forme (écriture, style, structure) devient ce qui permet de jouer avec un contenu" (p. 22); "ce n'est pas seulement (ni même peut-être principalement) son aventure intérieure" (p. 69); "la civilisation blanche (= capitaliste, moderne)" (p. 86); "un répertoire culturel (le Québec catholique des années cinquante) qui fournit d'emblée une matrice à l'imaginaire (p. 121); “Et c'est cette perte même (la conscience aiguë, affolée de cette perte)* (p. 183); "sous le signe de l'exil (psychique, fictif), du manque" (p. 201); "le métissage des cultures est non seulement travaillé par une double nostalgie (références françaises, culture yiddish d'Europe de l'Est)"(p. 205); "il se définit désormais comme épreuve, comme passage (de la mort à la naissance, du même à l'autre, de l'identique au changeant)" (p. 206).

28 "[...] certains poètes (Crémazie, Nelligan, Saint-Denys Garneau, Grandbois)" (p. 45); "(notamment chez Miron, Chamberland, Aquin)" (p. 67); "(exil égyptien chez Anne-Marie Alonzo, italien chez Caccia et d'Alfonso, pour ne citer que ceux-là.)" (p. 190). Les exemples proviennent aussi parfois du corpus à l'étude: "Les injures que Don Quichotte lui-même adresse à Steven ("un poète! un lettriste sans doute, un sans cour, un affreux [...], l'exilé putassier" [...J) ne font pas le poids : l'exilé putassier porte une auréole" (p. 129).

29 "Des musiciens (sinon des magiciens) du verbe [...] ou harpistes du verbe (ce qui n'empêche pas qu'ils puissent nous émouvoir et nous parler)" (p. 28); "des antagonistes qui obligent à prendre parti (à peu de nuances près, ce sera en faveur de Grandbois). (p. 45); "Crémazie et Nelligan l'avaient habité et subi dans la passivité (au moins pour le second, cette affirmation demeure évidemment discutable)" (p. 52); "la disparition des espèces (en l'occurrence ici : d'une espèce)"(p. 89); "Il y aura chez Aquin, Godbout, Blais, Ducharme le même mythe (je ne parle pas ici des différences)" (p. 104); "la "poésie" (ou disons plutôt, pour l'instant, une certaine poésie)" (p. 105); "le monde moderne (ou le monde québécois, mais l'un ici ne va pas sans l'autre). (p. 105); "c'est une lettre en vers (!) que le roman nous donne à lire" (p. 111); "une préoccupation centrale (je veux dire: explicite dans son œuvre). (p. 137); "après une entreprise (certes en partie réussie) de repaysement " (p. 189). 
134

les mots qu'il emploie et sur les énoncés qu'il avance; il agit en observateur et en commentateur de son propre discours. Ce procédé donne au locuteur la possibilité de se mettre en scène et d'esquisser le profil d'un allocutaire qu'il faut instruire. Il s'agit de construire un équilibre garant de l'adhésion continue de l'allocutaire tout en offrant au locuteur-essayiste la possibilité de combler ce double désir qu'il a de "décrire la réalité telle qu'elle est en elle-même et [d']imposer un point de vue sur elle" 30 .

\section{L'hétérogénéité}

La coprésence du discours auctoriel et du discours d'autres locuteurs met en scène l'hétérogénéité et pose la question de la distance du discours auctoriel par rapport aux discours "étrangers". Nous analyserons les marqueurs de l'hétérogénéité énonciative que l'on peut repérer notamment aux points d'insertion du discours rapporté, ou à des signes tels l'utilisation des guillemets et de l'italique. Eu égard au discours rapporté, l'importance quantitative, le type de textes cités, le degré de précision de la référence bibliographique, les occasions où il y a citation, les verbes destinés à introduire ce discours, la nature de la citation, etc., sont autant de phénomènes susceptibles d'être soumis à notre attention.

À lire l' "Avertissement ", on s'aperçoit qu'il y a d'entrée de jeu une double indexation de l'hétérogénéité : dans l'objet même du discours ("interroger dans les textes et les œuvres un certain mode d'être de la conscience québécoise [...] au sens où "mode d'être" signifie aussi "mode d'éclatement", et où l'un renvoie toujours à l'autre et au pluriel", p. 10), ainsi que dans l'énonciation peuplée d'une panoplie de co-énonciateurs. Nepveu mentionne six essayistes qui ont inspiré sa démarche et qui ont laissé des traces, explicites ou implicites, dans son ouvrage (p. 9); il nomme en outre Michel Morin et Claude Bertrand, à qui il emprunte l'expression "territoire imaginaire "; il reconnât enfin sa dette envers l'école herméneutique allemande, en particulier envers Gadamer, Jauss et Iser. Insérés dans l'«Avertissement", ses

30 Ces propos renvoient à ceux de Jean Terrasse pour qui l'essai littéraire est le produit d'une tension entre ces deux désirs apparemment contradictoires (Rbêtorique de l'essai littêraire, Montréal, Presses de l'Université du Québec, 1972, p. 129). 
remerciements évoquent encore six autres "collaborateurs". Le texte relève donc d'un locuteur distinct de tous les autres, mais portant en lui les traces de l'Autre.

Pour caractériser l'ouvrage de Nepveu sous l'angle du discours rapporté, quelques observations générales s'imposent dès une première lecture: il faut d'abord noter le très grand nombre de citations tout au long de l'ouvrage (citations dont la référence bibliographique complète est donnée dans les notes réunies à la fin) et ensuite souligner l'extrême diversité des énonciateurs, provenant de différents champs culturels. L'accumulation des citations provoque un effet d'érudition qui fait du locuteur une autorité en la matière (à titre de propriétaire d'un savoir) et un locuteur "autorisé", puisqu'il connât et manie le discours d'autrui. En substance, il s'agit d'un locuteur qui se présente comme étant digne de la confiance de l'allocutaire; la présence du discours citationnel prend largement appui sur sa vaste expérience. De fait, par la transcription de ses innombrables lectures, Nepveu assure sa propre légitimité en même temps qu'il suggère des axes de lisibilité permettant leur mise en relation; sous cet angle, L'écologie du réel participe pleinement du phénomène que Nicole Fortin a qualifié d' interlisibilité", au sens où l'ouvrage sollicite non seulement les œuvres du corpus mais surtout leur relecture critique ${ }^{31}$.

Sur la scène énonciative, le souci de précision de la référence permet de délimiter de façon non équivoque la parole d'autrui et de spécifier ce qui correspond au "non-moi du locuteur" (Maingueneau) ${ }^{32}$; en effet, dans cet imposant assemblage d'énonciateurs, le locuteur doit pouvoir se démarquer et constituer son propre espace de parole. Cette non-équivocité de la parole d'autrui produit, en creux, la non-équivocité de la parole du locuteur. Pạr ailleurs, il faut mentionner que si le discours emprunté à autrui est généralement très bien circonscrit et identifié, il n'en demeure pas moins que l'emprunt des concepts se fait le plus

31 "Interlisibilité " définie comme "une interrelation entre les œuvres qui ne provient pas de leur écriture [...] mais de leur relecture critique qui contribue à les inscrire dans des réseaux où ils deviennent interdépendants et "intersignifiants" ; Nicole Fortin, Une littérature inventée. Littérature québécoise et critique universitaire (1965-1975), Québec, Presses de l'Université Laval, coll. "Vie des lettres québécoises", 1994, p. 102.

32 Dominique Maingueneau, Nouvelles tendances en analyse du aiscours, Paris, Hachette, coll. "Hachette Université ", 1987. 
souvent sans trop de précisions concernant le contexte ou les présupposés théoriques. Ainsi, une notion aussi centrale que celle d'"écologie" n'est pas clairement définie; le lecteur sait que le concept est emprunté à Bateson, mais il ignore ce qu'il recouvre pour ce dernier. Son contenu notionnel demeure non précisé à la fois par rapport au texte d'origine et dans l'utilisation qu'en fait Nepveu, si bien qu'il fonctionne en partie sur un mode allusif, métaphorique. Autant les frontières sont bien définies entre les actes d'énonciation divers (et donc, comme on l'a vu, entre le sujet locuteur et son "non-moi"), autant elles demeurent incertaines lorsqu'il s'agit du contenu des concepts. Nepveu utilise une notion "validée", "cautionnée" par une autorité mais dont la définition demeurée floue lui laisse toute latitude pour la faire sienne. Nous examinerons plus loin l'usage de la notion de "territoire imaginaire".

Dans L'écologie $d u$ réel, le discours rapporté procède de divers types de discours, théorique, essayiste, critique, poétique et fictif. Pour expliquer cette diversité, il faut prendre en considération la visée de l'ouvrage, qui concerne à la fois l'histoire littéraire et l'étude de certains auteurs, chacun de ces aspects renvoyant à des usages particuliers du discours rapporté. Par exemple, les extraits du discours critique peuvent servir à tracer le portrait de la réception d'une cuvre ou à discuter d'autres interprétations de celle-ci. Mais il arrive que Nepveu use du discours rapporté d'une façon non conventionnelle et c'est ce que nous voudrions mettre en lumière. À titre d'exemple, voyons comment l'auteur, au chapitre III, utilise les divers types de discours rapportés en relation avec la notion d'exil. Il veut montrer le rôle de catalyseur qu'a joué la notion d'exil pour la littérature québécoise, notamment en rapprochant l'exil géographique de Crémazie et l'exil imaginaire - la folie - de Nelligan. Il cite le discours essayiste de Naïm Kattan (p. 47), qui a souligné le rôle fondamental de cette notion pour l'Occident européen; il cite également le discours de critiques littéraires (Marcotte, Aquin, De Grandpré et Vachon) portant sur l'exil de Crémazie et de Nelligan (p. 48-52). Nepveu ne s'intéresse pas seulement au discours sur l'exil, mais également au discours de l'exil dans les textes des poètes et des romanciers (Ducharme, Brossard, Beaulieu, etc.). Il va jusqu'à citer le discours - et c'est là qu'il est non conventionnel - qu'un personnage romanesque (ducharmien) tient sur Nelligan: Mille Milles, au même titre que les théoriciens, les 
essayistes, les poètes, va relater "son" Nelligan. Tous ces discours sont situés à un même niveau par rapport au discours citant; Nepveu les accumule comme autant de preuves.

L'intertexte est donc constitué d'un mélange des discours fictionnel, critique et essayiste. Une des conséquences de ce rapprochement est l'effacement de la hiérarchie entre le discours de la réalité et celui de la fiction. Ainsi, au chapitre VI, Nepveu examine la relation conflictuelle entre poésie et roman chez Gilles Hénault, Paul Chamberland... et Jean Le Maigre (le personnage d'Une saison dans la vie d'Emmanuel de Marie-Claire Blais), ce faux poète devenu romancier qui raconte sa propre histoire. Nous avons vu auparavant que Nepveu situait dans un même espace imaginaire et son propre discours d'essayiste, et la parole des poètes; nous retrouvons, dans cette réunion des discours du poète fictif et des poètes "réels", la même nécessité de ce lieu (imaginaire) de rencontre de tous les discours. En somme, la pluralité des voix (fictives, théoriques, critiques, etc.) qui se partagent la scène énonciative, reliée à la présence de multiples citations et à la convocation de divers types de discours, contribue à valider le propos par l'accumulation de témoignages qui, tous, tiennent le même discours, disent la même chose. Il y a très peu de voix discordantes dans L'écologie du réel; c'est la concordance des innombrables paroles qui convainc.

Les citations placées en exergue se présentent différemment. Notons d'abord qu'il s'agit surtout de citations d'auteurs québécois (à l'exception de celles de Nietzsche et de Pasolini), qu'elles sont au nombre de 14 en tête de 8 des 12 chapitres et qu'elles sont identifiées par le seul nom de l'auteur, sans précision de l'ouvrage d'origine. Ce discours cité occupe une position privilégiée, car il instaure un dialogue avec l'ensemble du chapitre. Toutefois, ce n'est pas toujours le contenu du texte cité qui fait ce lien; assez souvent, c'est la présence d'un signifiant en particulier, signifiant qui se retrouve en plusieurs endroits du texte et même dans le titre. Ainsi, le chapitre III, intitulé "L'exil comme métaphore", s'ouvre sur deux épigraphes, l'une comportant le mot "exil" et l'autre le mot "métaphore". Le chapitre IV, dont le titre, "Un trou dans notre monde", est une citation de Saint-Denys Garneau, met en exergue ce passage de Beausoleil: "à rien une manière de trou oui un trou qui tourne sur place lentement qui tourne sur lui-même en plein vide..."; l'étude explore les diverses facettes du vocable "trou" et se clôt sur ce mot. Placée en tête 
138

de chapitre, l'épigraphe se situe donc dans un lieu où la parole citée est davantage libérée du discours citant; ce sont les discours de deux auteurs qui se rencontrent et se réfléchissent, celui de la citation et celui, sous-entendu, du discours citant. Soulignons enfin que les épigraphes ont ultimement pour fonction de cautionner le propos de l'ouvrage, en donnant à voir et en commentant l'existence d'une littérature québécoise, morte, naissante ou post.

L'italique et les guillemets - marqueurs par excellence de l'hétérogénéité, dans la mesure où ils permettent l'inscription de l'autre et de l'ailleurs du discours ${ }^{33}$ — pullulent dans le texte de Nepveu. Un survol rapide permet d'obtenir un éventail sinon exhaustif, du moins représentatif de l'utilisation de l'italique. Mentionnons d'abord l'abondance des titres de livres et des noms de revues, tous en italique. Hormis ces cas, l'italique est utilisé pour mettre l'accent sur un mot et en marquer une nuance ou une intensité particulières ${ }^{34}$. Il permet également de souligner un certain nombre de termes-pivot; ce type d'utilisation se retrouve principalement au début et à la fin de l'ouvrage ${ }^{35}$, lieux stratégi-

33. Sur cette question de l'hétérogénéité énonciative, voir Jacqueline AuthierRevuz, "Hétérogénéité(s) énonciative(s)", Langage, $n^{\circ} 73$ (mars 1984), p. 98 111, et Maingueneau, op. cit., p. 53-94 (soit le chapitre II, "L'hétérogénéité ").

34 On retrouve notamment : “le regard qu'il pose sur la nature se caractérise justement par le fait qu'il s'agit d'un regard" (p. 27); "le sujet [...] est moins substance que désir de substance" (p. 31) ; "dans la mesure où c'est naturellement (et non intentionnellement) que le forme artistique devrait [...] tra duire la richesse ontologique" (p. 33); "Imaginaire signifie ici [...]. Imaginaire signifie que" (p. 35); "C'est Jean-Louis Major, je crois, qui s'étonnait de constater qu'à peu près aucun poème de Garneau ne lui paraissait entièrement satisfaisant " (p. 36); "il découle à la fois du ton familier [...] et de l'enjeu existentiel lui-même" (p. 39); "que la poésie doit échouer si elle prétend dire vrai" (p. 755); "Mais le pluralisme fort expose les différences, il les mesure et les interroge" (p. 215). Voir aussi les pages 93, 166, 176, 187, 199 , 204 et 216.

35 Voici la liste, remarquablement longue, des mots en italique dans la première section du premier chapitre (p. 13-19): "l'expression: littérature québécoise"; "lui donner un nom: l'institution"; "un autre crépuscule: la fin de la littérature québécoise"; "à parler désormais, avec un certain à-propos, d'une littérature post-québécoise"; "c'est l'écriture qui s'est peu à peu imposéen; "que je suis (que nous sommes)"; "La littérature "québécoise", elle, transforme ou veut transformer la réalité ". Dans les dernières pages, on retrouve: "participe d'une véritable écologie de l'ici. (p. 209); "Écologie de l'ici: aménagement, gestion des ressources" (p. 210); "1. L'estbétique de la fondation [...]. 2. L'estbétique de la transgression [...]. 3. L'estbétique de la ritualisation [...].v (p. 212). 
ques où il convient d'insister sur certains mots ou notions dont la portée s'étend tantôt à ce qu'il faut retenir pour la lecture qui va suivre, tantôt à ce qu'il faut retenir une fois la lecture terminée.

L'effet de démarcation créé par l'italique semble avoir davantage pour but de marquer l'intensité, l'intonation, à la façon d'une modulation interne, que de signifier un extérieur du discours, comme dans le cas de mots étrangers. Ces mots en italiques sont en effet ceux auxquels le locuteur a donné une inflexion particulière. Cela constitue-t-il pour autant une marque de subjectivité? Oui, car en dépit du recours à des tournures impersonnelles et en l'absence du pronom je, le locuteur, par ces indications construites spécialement pour l'allocutaire, signale malgré tout sa présence en tant qu'instance énonciative.

Les guillemets sont aussi très nombreux dans L'écologie du réel. Parfois, ils indiquent que le locuteur utilise un mot qui n'est pas sien sans pour autant en préciser l'origine; cette énonciation autre mais incertaine doit être attribuée à un autre registre discursif, à un discours particulier, à un énonciateur vague, etc. Ainsi en va-t-il des expressions suivantes: "ne pas être "la plaie et le couteau" (p. 17), "un "faire-moderne" (p. 54) ou encore "l' insensé" féminin" (p. 167). Mais chez Nepveu, les guillemets sont surtout des marques de citations dûment attribuables à un autre locuteur; de nombreuses citations courtes sont ainsi insérées dans le texte, et d'ordinaire à l'intérieur de la phrase ${ }^{36}$; bien souvent, elles correspondent à l'emprunt d'un seul mot ou d'une expression. La référence se résume parfois au seul nom de l'auteur, qui apparaît entre parenthèses ou est introduit par une locution $^{37}$; parfois elle est inscrite dans les notes. Il s'agit donc

36 Par exemple: "La force de Miron est d'écrire l'exil comme l'aventure psychique totale d"un homme debout qui s'insère/dans la fêlure de sa vie" " (p. 56).

37 "André Laurendeau parlait [...] d'une "frénésie d'auto-critique" " (p. 17); "la lucidité des "Nouveaux Québécois" de 1960"(p. 17); "depuis les pages irréfutables consacrées par Gilles Marcotte à la "prose" de Rimbaud" (p. 28); " la "sainteté" dont Emmanuel Lévinas nous rappelle qu'elle est [...] synonyme de séparation" (p. 28); "une "mode" au sens où l'entendait Baudelaire" (p. 30); "ce que Garneau lui-même appelait le "lyrisme coulant" (p. 33); "cette "communauté inavouable" dont parle Blanchot" (p. 38-39); "cette "apparition" dont parle Rivard" (41); "Garneau ne s'approche de la poésie "que pour lui manquer" selon la formule bien connue de Georges Bataille. (p. 41); "nudité qui est aussi l'“ insoutenable légèreté de l'être" dont parle Kundera" (p. 48); "comme si un rapport "sain" (Hubert Aquin) [...] pouvait 
d'un fragment de discours exogène qui est introduit et utilisé - à la fois mention et usage - par le discours citant. Impressionnante, la quantité de citations de mots (ou expressions) "d'auteurs" révèle l'attention qu'accorde Nepveu à la matérialité des mots. Une telle pratique permet de convoquer plusieurs locuteurs sur une petite surface textuelle et de construire une situation énonciative où les voix des locuteurs se rejoignent par le truchement de mots partagés. Elle donne de l'amplitude au discours de Nepveu, qui se présente non seulement comme maitre d'un savoir, mais également comme maître d'un savoir-faire dans l'art de rapprocher les discours, qu'il s'agisse d'accorder le sien à celui des autres ou d'harmoniser ces derniers entre eux. Parce qu'il reprend les mots ou expressions au fil des pages ou des chapitres, Nepveu réussit à construire à travers ces déplacements un réseau d'appartenance; c'est dans ce trajet qu'il apprivoise le mot de l'autre et que ce mot devient de plus en plus le sien.

Examinons par exemple cette citation: "École de vertige", l'exil devient du même coup une école de "la sensation vraie" "

constituer la norme littéraire" (p. 49); "écriture de "mots-flots" (Giguère)" qui se déverse comme un immense désir (p. 52); "du pléthorique et (du) lacunaire" dont parle Gérard Bessette " (p. 58); " la coulée triomphale" (Aquin) qui assurerait le salut" (p. 69); "ce que Freud appelle "l'inquiétante étrangeté" " (p. 92); "même à "l'état de cétritus" (Miron), le sujet s'affirme" (p. 101); "au "territoire imaginaire" de la culture québécoise, pour reprendre la formule de Michel Morin et Claude Bertrand" (p. 114); "ce "projet romantique" dont a parlé Jacques Michon" (p. 129); "son "entrée dans la modernité", pour reprendre l'expression de Marcel Fournier" (p. 137); "comme "défondation" pour reprendre le thème que Gianni Vattimo [...] développe " (p. 144); "Bored but hyper", pour reprendre l'expression d'Andy Warhol" (p. 149); "ce qui s'ouvre sur une nouvelle "écologie de l'esprit", pour reprendre l'expression de Gregory Bateson" (p. 152-153); "ce que Gregory Bateson appelle la "rationalité objective" (p. 154); "à ce qu'on pourrait appeler, à la suite de Derrida, un certain "ton apocalyptique" " (p. 155); "la "raison baroque" qu'a analysée Christine Buci-Glucksmann" (p. 160); "comme "overdose d'analogies", pour reprendre l'expression de Villemaire dans La vie en prose" (p. 165); "la "fatigue culturelle" dont parlait Aquin en 1962 refait surface" (p. 169); "pour reprendre l'expression d'Élise Turcotte, d'une "mémoire de l'oubli" (p. 186); "dans ce que Charron appelle "la blancheur du non-agir", rejoignant en cela exactement l'idée de cette "communauté désœuvrée" dont a parlé Blanchot " (p. 190); "celle du "rhizome" dont ont parlé Deleuze et Guattari " (p. 194); "la nouvelle culture québécoise [...] "recueillante", un peu selon la définition qu'Heidegger donnait du langage dans son célèbre entretien avec un Japonais, dans Acbeminement vers la parole. (p. 217); et ainsi de suite. 
(p. 204). Les expressions entre guillemets ne sont pas expressément attribuées à un locuteur, mais elles l'ont été précédemment; Nepveu a cité à deux occasions les paroles de Cioran ${ }^{38}$ et l'expression "la sensation vraie" est reprise du chapitre précédent, intitulé "L'ère de la sensation vraie". Cette expression est d'abord revendiquée par le locuteur, la présence du pronom je en faisant foi : "une époque, que j'appelle "l'ère de la sensation vraie" " (p. 191); puis Nepveu y revient pour en indiquer la provenance: "Le nom que je donne à cette "ère" fait écho au roman de Peter Handke, L'beure de la sensation vraie" (p. 191). Dans les pages qui suivent, la formule revient trois fois sous la forme "l'ère de la sensation vraie" (p. 192-195) et deux fois sous celle de "sensation vraie" (p. 193). Nepveu a fait converger son discours et celui de Peter Handke; il associe l'expression de ce dernier à d'autres images, celles de la danse, de l'ange, de l'âme et du "rhizome" toutes références créant, au sein de son propre texte, un réseau entre De Bellefeuille, Wim Wenders, Buci-Glucksmann, Émile Nelligan, Paul-Marie Lapointe, Rilke, Deleuze et Guattari. Forte de cette alliance et ainsi devenue le signe de ralliement d'une "école", l'expression rejoint l'“ école de vertige" de Cioran.

Une autre expression chère à Nepveu est celle de "territoire imaginaire". Elle fait référence à une notion élaborée par Michel Morin et Claude Bertrand à la fin des années 1970 39. On la retrouve dans l'épigraphe initiale empruntée à ces derniers ${ }^{40}$, puis dans l' Avertissement " 41 et à divers autres endroits du texte (dont les pages 112 et 114). L'épigraphe permettant d'associer le discours nouveau à d'autres discours qui le précèdent, Nepveu instaure donc d'entrée de jeu une parenté entre son travail et celui de Morin et Bertrand. La citation choisie permet également

38 "Avantages de l'exil", écrit sardoniquement Cioran. "L'exil, à ses débuts, est une école de vertige" (p. 57); "Dans un court essai auquel je me suis souvent référé, "Avantages de l'exil", Cioran (lui-même immigrant roumain en France) mettait en lumière l'intensification de l'imaginaire que suscite la position d'exilé (l'exil comme "école de vertige")" (p. 200).

39 Morin et Bertrand, op. cit.

40 "Ce surgissement du territoire imaginaire, indissociable de l'émergence du mythe, implique cette possibilité du déplacement de la distance par rapport au territoire réel, et en même temps, la mise en rapport de celui-ci avec d'autres territoires, à la fois réels et imaginaires. "(p. 8).

41 "[...] dans la mesure où l'imaginaire traversant ces figures élabore une véritable "écologie du réel", configure ce "territoire imaginaire" dont ont parlé Michel Morin et Claude Bertrand" (p. 10). 
142

de relier l'imaginaire du "territoire" (énonciation de l'autre) au réel de l' "écologie" (énonciation de Nepveu). Le critique récupère l'expression "territoire imaginaire" comme si elle était extraite de la citation d'ouverture, alors qu'en réalité elle constitue la notion centrale de l'ouvrage de Morin et Bertrand, ouvrage dont la présentation est retardée jusque dans une note du chapitre VI. Dans ce sixième chapitre, on rencontre l'expression à trois reprises: la première fois, elle est entre guillemets et ne semble renvoyer qu'au discours antérieur (p. 112); la deuxième fois, une note précise la référence bibliographique complète de l'ouvrage (p. 114); enfin, l'expression apparaît une troisième fois, sans guillemets, totalement insérée dans le fil du discours (p. 114). Dans chacun de ces cas, une relation différente s'inscrit entre le locuteur et cet emprunt, qui va de l'altérité la plus totale - celle de l'épigraphe - à une prise en charge complète dans un discours qui efface les marques d'hétérogénéité que sont les guillemets.

Ainsi Nepveu, à travers ces jeux avec les mots des autres, conjoint à son travail d'essayiste l'activité de l'écrivain, cet amoureux des mots qui est toujours un peu "voleur de mots", comme le soulignait Michel Schneider ${ }^{42}$.

\section{La fascination de la parole}

Les stratégies énonciatives déployées dans L'écologie du réel reconduisent la définition littérale de l'écologie par le maintien des axes subjectif et polyphonique, axes qui construisent la figure d'un lecteur en constante interaction avec son milieu. En mettant en scène et en intégrant à son propre discours le déjà dit de la critique littéraire, Nepveu accuse à la fois la perméabilité de sa parole et l'ampleur du territoire discursif parcouru. L'absence de confrontation - la polémique est rare - rend encore plus manifeste la conception de la littérature qui informe l'essai: espace de dialogues et de lectures croisées, le littéraire est ici mouvant, libre, ouvert, indéfiniment malléable.

De par son caractère systémique, l'entreprise de Nepveu sanctionne encore une redéfinition de l'acte critique. D'une part, le quadrillage minutieux du champ littéraire, outre la mise au jour de convergences inusitées, affirme la nécessité de la prise en con- 
sidération de l'ensemble, déplaçant de ce fait la visée cognitive de la lecture. La saisie du sens, dans cette perspective, s'ordonne non seulement à l'élucidation du texte, mais fonde sa pertinence sur son aptitude à reconnaître, ou à créer, des réseaux discursifs susceptibles de le rendre signifiant. D'autre part, l'indifférenciation des référents critiques et fictifs donne à voir, en filigrane, l'ordre interprétatif qui gère la démarche de Nepveu. Cet ordre selon lequel tous les savoirs ne sont que des interprétations engage la conception du réel - et le propos est bien celui d'une écologie du réel -, réel qui se révèle hautement tributaire des possibles discursifs. En ce sens, l'acte de lecture, chez Nepveu, est indissociable de l'inscription de cet acte, voire de son esthétisation.

Un tel décloisonnement des frontières entre le dire et le déjà dit, entre le réel et le fictif, entre la critique et l'écriture, signale certes une singularité énonciative. Le critique, ici, se fait le relais de la parole des autres, recyclant de larges pans de l'édifice littéraire, balisant autrement ce territoire imaginaire. S'agit-il pour autant d'un changement de paradigme? À la fois évaluative, descriptive, interprétative, la parole de Nepveu est avant tout jubilatoire et la littérature, vivante de tous ses mots, devient un acte de construction, d'appropriation du réel. 